\title{
ANTON BIERL \\ Alt und Neu bei Aristophanes \\ (unter besonderer Berücksichtigung der Wolken)
}

\section{Einleitung}

Das Streben nach Neuem, der Ehrgeiz, Altes hinter sich zu lassen, ist in jeder Kultur ein wichtiges Faktum." Während das Neue in der Neuzeit schließlich zum Konstituens wurde, so daß man alle Änderungen stürmisch als Fortschritt preist, ${ }^{1}$ stand man in vielen Epochen dem Neuen eher skeptisch gegenüber, da es Althergebrachtes notwendigerweise entwertet. Gerade die Moderne zeichnet sich durch ein Hervorbringen von innovativen Wenden im geistigen und kulturellen Leben aus, wobei man aber meist übersieht, daß in dem Bestreben, das Revolutionäre und Noch-nicht-Dagewesene herauszustreichen, alles Vorausliegende undifferenziert zusammengezogen wird. ${ }^{2}$ Alt und Neu befinden sich permanent in einer gegensätzlichen Spannung. Reformen werden durch die Einverleibung von Altem legitimiert und geadelt, so daß Neues oft als nichts anderes als das Alte in verändertem Gewande erscheint. Die Wirkweisen dieser Dialektik sind mannigfach. Gerade in der Kunst, Kultur und Literatur pflegt man die Fähigkeit zur Innovation schon seit jeher hervorzuheben. Selbst in der Antike, wo das Neue eher im Sinne von Unerhörtem und Umstürzlerischem pejorativ besetzt ist, wird der Anspruch auf Originalität auf dem Feld der Künste schon seit den Anfängen von allen betont.

Jeder Dichter will sich gerade in einem Gattungskontext, der noch dazu wie im Falle des griechischen Dramas vom Agonalen bestimmt ist, von seinen Vorgängern und Rivalen absetzen sowie die generischen Grenzen spielerisch austesten. Denn nur derjenige, der Aufsehen erregt, hat eine Chance, den Wettkampf zu gewinnen.

* Der Aufsatz ist den zahlreichen Neuerern der Universität Basel gewidmet. Ich danke den Mitsymposiasten für die kritischen Diskussionen und Anregungen, insbesondere dem Kollegen von Ungern-Sternberg für die vielen Ermunterungen.

1 Vgl. Meier 1980, 435-443.

2 Vgl. Schmitt 2002 und Moog-Grünewald 2002. 


\section{Neuheit und ihre Grenzen in der Alten Komödie}

Im Falle der Alten Komödie ist die Thematik des Alten und Neuen besonders relevant und sinntragend. Zum einen stellt auch Aristophanes, der einzige Verteter der Gattung, von dem wir nicht nur Fragmente, sondern elf vollständig erhaltene Komödien überliefert haben, immer wieder das Innovative seines Theaters heraus.

Entgegen den häufigen Ankündigungen, auf den vulgären Humor der Volksposse zu verzichten, setzt Aristophanes just diesen immer wieder ein. ${ }^{3}$ In der Pose des gebildeten Poeten distanziert er sich wiederholt von der volkstümlichen Farce, deren sich seine Rivalen bedienen, indem er öffentlich erklärt, vom

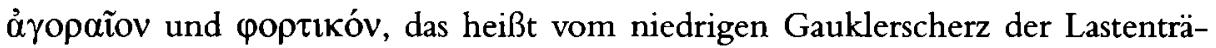
ger auf dem Marktplatz, Abstand zu nehmen und eine literarisch gehobene Komödie verfassen zu wollen. So wendet er sich beispielsweise in den Wespen (V. 56-66) mit folgender Erklärung an sein Publikum:
$\mathrm{Zu}$ Großes dürft ihr nicht von uns erwarten,
Doch auch nicht Spaß, aus Megara gestohlen:
Es kommen keine Sklaven, die aus Körben
Mit Nüssen werfen nach dem Publikum,
Kein Herakles, den man ums Essen prellt;
Euripides wird nicht erneut gerupft,
Selbst Kleon, hat er auch geglänzt, von neuem
Woll'n deshalb wir ihn nicht zu Brei verrühren.
Wir bringen euch ein Lustspiel, das Verstand hat,
Nicht eben mehr als ihr, doch mindestens
Gescheiter ist's als manche plumpe Farce.

Doch holt der Dichter diese dummen Späße paradoxerweise gleich wieder zur Hintertür herein und integriert sie in sein komisches Spiel; offenbar braucht er die alten Zoten, um durch Lachen das Publikum einzubeziehen. ${ }^{4}$

Dem Genre sind eindeutige rituell-pragmatische Grenzen gesetzt. ${ }^{5}$ Aristophanes befindet sich an der Schwelle von einer dominant mündlich geprägten zu einer weitgehend schriftlich bestimmten Kultur der Rezeption. Die Alte Komödie ist weniger substantielle Literatur als vielmehr in ihrer Funktion und ihrem Sitz im Leben zu verstehen. Die Theaterauffuihrungen sind bekanntlich in einen rituellen Rahmen zu Ehren des Gottes Dionysos eingebettet. In seinem Umfeld treten mit Vorliebe Verkehrungen und Verzerrungen des altäglichen Horizonts auf. In einer oral geprägten Lebensform ist das Denken weitgehend durch den Mythos und das

3 Vgl. Ar. Nub. 537-562; Pax 739-751; Vesp. 57-66; Ran. 1-18. Zu diesen Äußerungen gegen die vulgäre traditionelle Farce vgl. Halliwell 1991, 290.

4 Zur Farce vgl. MacDowell 1988 und Lowe 1988. Zum Spielcharakter des komischen Tanzes vgl. Bierl 2001, 86-96.

5 Vgl. zum folgenden Bierl 2002b. 
Ritual einer eng begrenzten Örtlichkeit, in unserem Fall Athen, definiert. Das aus rituellen Schwarmumzügen hervorgegangene Spiel, das die heitere Verkehrung aller Normen im Rückfall in eine vorzivilisatorische Stufe feiert, wurde erst relativ spät (487/86 v. Chr.) in die theatralen Agone der Polis integriert und ist in viel größerem Maße als die Tragödie Ritual geblieben. ${ }^{6}$ Die Funktion der komischen Gattung besteht also darin, im komischen Sprung ${ }^{7}$ zurück in atavistische Zeiten aus der nach unten pervertierten Perspektive des Anderen, Häßlichen und DerbObszönen eine komplementäre Sicht auf die aktuelle Welt und ihre Gesetzmäßigkeiten zu ermöglichen. ${ }^{8}$ Der als Ganzkörpermaske ${ }^{9}$ agierende komische Held begibt sich dabei meist auf eine Reise in eine Anderwelt, die auf den realen Alltag und die Polis dialektisch bezogen bleibt. Aus diesem zeitlich und örtlich so ganz anderen Territorium bezieht er Kraft und Heilpotential. Dieses überträgt sich in der Identifikation auf das Publikum, das auf der Folie der heiteren Alterität gleichzeitig Angebote für die Identität erhält. ${ }^{10}$

Die funktionelle Gattungsbestimmung impliziert ein relativ stabiles, festumgrenztes Gerüst, das stets auf andere Weise ausgefüllt wird. ${ }^{11} \mathrm{Neu}$ ist die Alte Komödie nie in dem Sinne, daß ihre Autoren vollkommen andere Wege gehen und in völliger Freiheit Handlungen erfinden. Sobald man die Grenzen mißachtet, geht sie ihrer generischen Funktion verlustig, was dann in der Neuen Komödie eines Menander in einer nun schriftlich dominierten Kultur geschieht. Eine Novität stellt also nur dar, wie ein Vertreter der alten Gattung den Vorgaben genügt. Das Andere kann auf Frauen, Tiere, Sklaven, Barbaren, ja sogar zeitgenössische Intellektuelle, Philosophen und tragische Dichter projiziert werden. ${ }^{12}$ Unerwartet und originell ist vor allem die Maske, die Art und Ausstattung des Chores sowie die gesamte öffentliche Performance als opsis.

\section{Das Neue und Originelle:}

\section{Aristophanes zwischen Mündlichkeit und Schriftlichkeit}

Gerade die Komödie braucht das Neue, das Unerwartete und Improvisierte des Einfalls für die Provokation des konstitutiven Lachens. ${ }^{13}$ Die Selbstdarstellung

6 Vgl. Bierl 2001, $29 \mathrm{f}$.

7 Vgl. Lohr 1986, 63-68.

8 Zur Perspektive des Häßlichen vgl. Brelich 1975, 112. Zum funktionell-komplementären Verständnis der Komödie, die mit einem ,Zurück zu den Anfängen operiert, vgl. Münz 1998, 78, 101, 109, 118, 134-136, 151f. und $228 \mathrm{f}$.

9 Vgl. Münz 1998, 109, 120, 132 und 275-279.

$10 \mathrm{Zu}$ diesen Ausführungen vgl. auch Bierl 2002a, $172 \mathrm{f}$.

11 Zu diesem Absatz vgl. Bierl 2002b. Vgl. auch Grilli 2001.

12 Vgl. Bierl 2001, 96-104.

13 Vgl. Lohr 1986, 175: „Zur Auffuhrungssituation der theatralischen Form ,Komödie“ 
des auf Originalität pochenden Dichters hat häufig in der Parabase ihren Ort: ${ }^{14}$

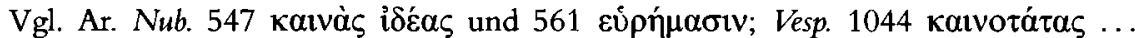

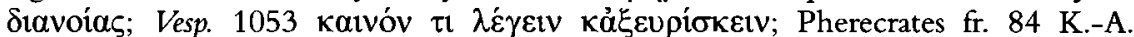

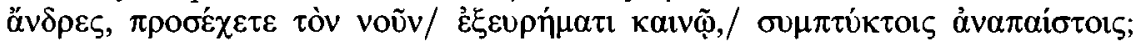

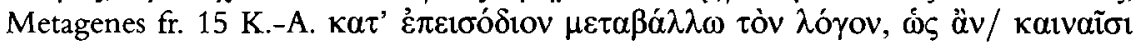

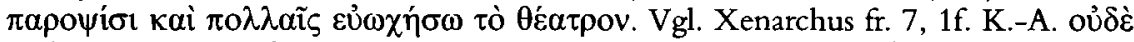

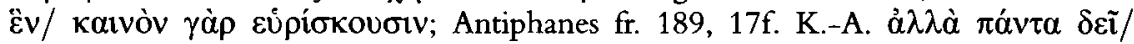

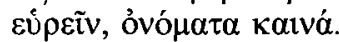

Die Äußerung des Dichters stellt freilich nur eine Abwandlung oder Weiterentwicklung der Eigenwerbung seines Chores dar, der seine besondere Qualität im Komödienagon zur Schau stellt. ${ }^{15}$

Das Insistieren auf Neuartigkeit hängt zum Teil mit dem Aufkommen einer dominanten Schriftkultur zusammen und wird dann für den Hellenismus typisch. ${ }^{16}$ Doch besteht der Topos schon seit der archaischen Zeit:

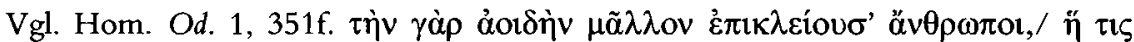

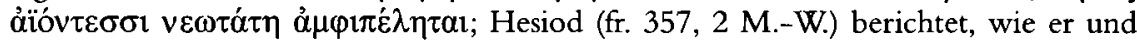

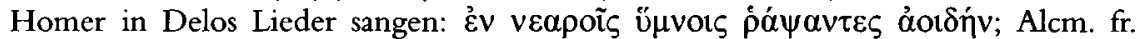

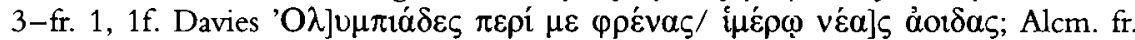

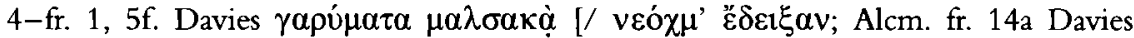

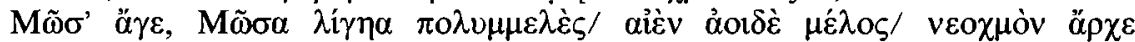

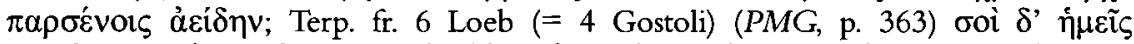

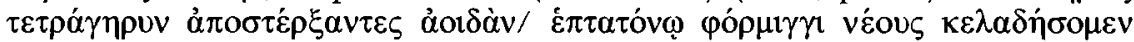

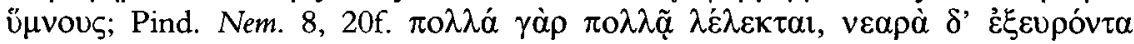

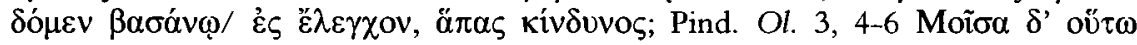

gehört immer ein Moment unvorhergesehener Neuheit, mit dem sich die Schauspieler für den Zuschauer meist nicht bemerkbar - den Raum der komischen Communitas schaffen; gerade auch in einem durchinszenierten Stück des modernen Repertoire-Theaters.“

14 Vgl. auch Sifakis 1971, 39. Außerhalb der Parabase im Prolog: Ar. Pax 54f. ó $\delta \varepsilon \sigma-$

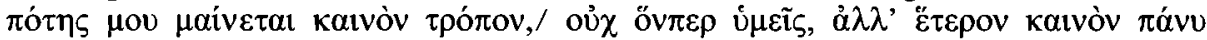
und andere Stellen ohne Bezug auf kalvó $\zeta$ bei Sifakis 1971, 39.

15 Zur Identifikation des Dichters mit dem Chorfuhrer (so beispielsweise in $N u b$. 518-562, Pax 734-764) vgl. Dover 1972, 50-53 und Sifakis 1971, 52. Die Anapäste können ebenso häufig für die Selbstdarstellung des Chores wie für die des Dichters verwendet werden; vgl. Hubbard 1991, 20. Der komische Chor kann unterschiedliche Stimmen auf sich vereinigen; gerade in der Parabase kann das chorische ,Ich $\%$,Wir" historischautobiographisch (als Dichter), allgemein politisch (als Chor in seiner lebensweltlichen Funktion), performativ (als Chor in der Rolle oder Funktion) und dramatisch (als Chor in der fiktionalen Rolle) vor das Publikum treten; der komische Chor kann in mehreren dieser Stimmen gleichzeitig reden und zwischen diesen Sprechhaltungen frei schwanken. Vgl. dazu Bierl 2001.

16 Vgl. Bing 1988, 22f. Zur Betonung der Neuheit in der hellenistischen Poesie vgl. Parsons 1993, 163-166 und den Beitrag von Ambühl (in diesem Band). 


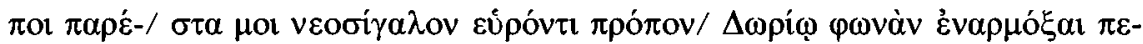

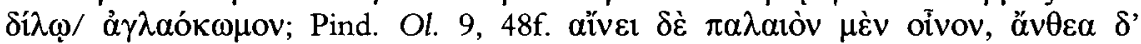

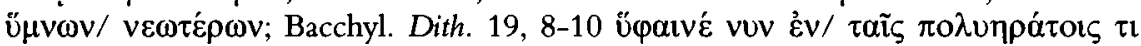

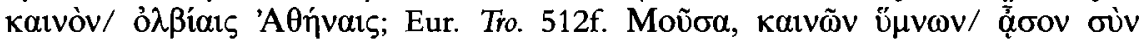

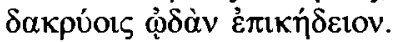

Nach Pindar und Aristophanes ist bei Timotheos, Kinesias und Pratinas ${ }^{17}$ parallel zur totalen Verschriftlichung eine Radikalisierung in der Betonung des künstlerischen Selbstbewußtseins zu beobachten, die in die Praxis des Hellenismus mündet. Die Alexandriner fassen die Neuheit dann vor allem in Kategorien des Buches. ${ }^{18}$

Aristophanes steht hier also am Ende des fünften Jahrhunderts v. Chr. deutlich an der Schwelle zu einer neuen Auffassung, wenngleich seine Verwendung des Anspruchs auf Originalität noch ganz im Okkasionellen aufgeht.

\section{Das Neue im fünften Jahrhundert v. Chr.}

Während die griechische Gesellschaft bis zum Anfang des fünften Jahrhunderts vergleichsweise stabil und neuerungsresistent war, kann man, wie insbesondere Christian Meier gezeigt hat, dann gerade in Athen, dem Ort der dramatischen Auffuhrungen im Dionysostheater, von einem ungeheueren Anwachsen von Fähigkeiten sprechen. ${ }^{19}$ Eine nie gesehene Aufbruchsstimmung und Dynamisierung in allen Bereichen des Lebens, insbesondere in der Politik und der Kultur, sind auf einmal zu konstatieren. Die Athener galten schließlich als notorische Neuerer. ${ }^{20}$ Das plötzlich empfundene „Könnens-Bewußtsein“ revolutioniert das Dasein. Allerdings geschieht die Entdeckung des Neuen immer in Abgrenzung vom Alten, so daß in dieser Epoche undifferenzierte Fehlurteile ihren Anfang

17 Bei Timotheos fr. 796 PMG tritt dieses Motiv besonders deutlich hervor: oủk

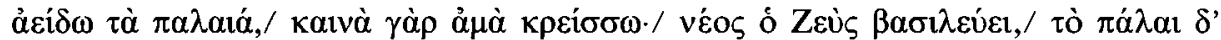

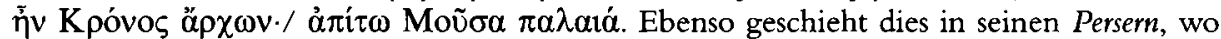
er (fr. 791, 211f.) beschreibt, daß die Spartaner ihn zur Rechenschaft ziehen, weil er ,die

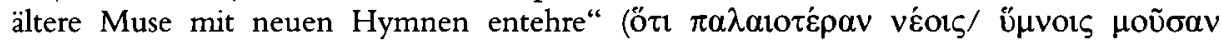

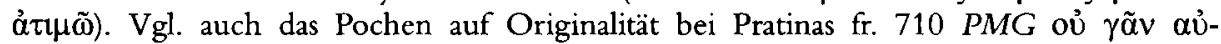

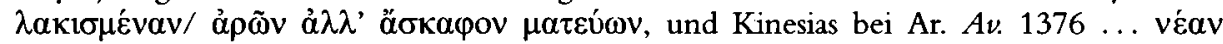

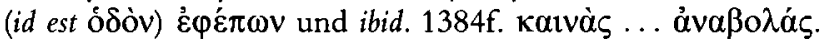

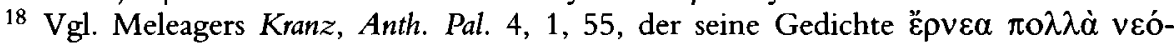

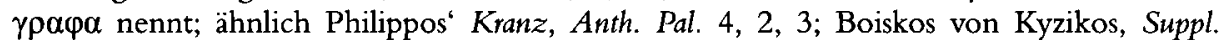

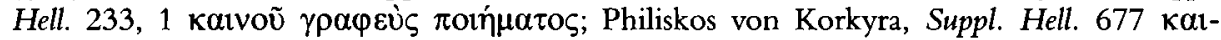

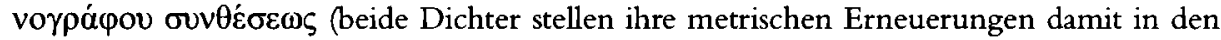
Vordergrund).

19 Vgl. Meier 1980, 435-499, bes. 469-499.

20 Vgl. Meier 1980, 447-450 und Manuwald 2000, 92 Anm. 60 (mit Belegen). 
nehmen. Die Zunahme von Wissen und die neuen Formen der Fragestellungen haben zur Folge, daß bei den Vertretern der Philosophie der Anspruch aufkommt, man lasse den Mythos radikal hinter sich und bewege sich auf den Logos zu. Diese Selbstaussagen wurden dann von der modernen Forschung als Geistesbewegung unter dem Motto „Vom Mythos zum Logos“ zusammengefaßt. ${ }^{21}$ Erst seit kurzem sieht man, daß diese Vorstellung bei aller vermeintlichen Tragweite zu kurz greift und sich beide Konzepte auch in dieser Epoche gegenseitig bedingen. ${ }^{22}$ Das heißt, Mythos und Ritual blieben trotz gegenteiliger Beteuerung weiterhin zentrale Diskurse der Polis.

\section{Alt und Neu auf der Inhaltsebene des Aristophanes}

Aufgrund des festen Verlaufsschemas der Alten Komödie, nach dem der Held in eine atavistische Anderwelt fällt, um eine komplementäre Sicht auf die Gegenwart zu eröffnen, erscheint gerade die Opposition zwischen Alt und Neu, bzw. zwischen einem Einst und Jetzt, auch in der Anlage der komischen Plots besonders prominent. ${ }^{23}$

Hinsichtlich der Verankerung im Mythos und Ritual kristallisiert sich hinter den einzelnen Stücken ein übergreifendes Strukturmuster heraus: die Alte Komödie verarbeitet in ganz betonter Art und Weise kultische Bestandteile von Ausnahmefesten. In ihnen setzt man die normale Ordnung für kurze Zeit außer Kraft, um dann mit frischem Elan und Optimismus zum gewohnten Kosmos zurückzukehren. Die Tempel der olympischen Götter werden geschlossen. Tabus, die sonst gelten, werden temporär gebrochen, bestehende Hierarchien auf den Kopf gestellt und die Menschen in eine mythische Phase des längst überwundenen Chaos versetzt, die von der Auseinandersetzung zwischen zwei Göttern oder ganzen Göttergenerationen geprägt ist. Schließlich wird die Welt in der Vergegenwärtigung der Schöpfungsgeschichte gleichsam wieder neu geschaffen. ${ }^{24} \mathrm{Im}$ offiziellen attischen Kult zeichnen sich besonders die dionysischen Anthesterien durch die beschriebene Charakteristik aus. Die Kronia, Haloa, Thesmophoria und die Skira sind ebensolche Übergangs- und Ausnahmefeste der verkehrten Ordnung. ${ }^{25}$

$21 \mathrm{Vgl}$. Nestle 1940.

22 Vgl. Most 1999; vgl. auch Buxton 1999 und Bierl (in Vorbereitung).

23 Vgl. auch Silk 2000, 379, 383, 392 und 423 (zum Motiv ,once but now").

24 Vgl. Auffarth 1991 und Auffarth 1994, der auch die kulturelle Abhängigkeit dieses Modells vom Alten Orient betont.

25 Zum Ausnahmefest der Verkehrung der normalen Ordnung vgl. Versnel 1993, 89-135, 136-227 und 228-288 (,Kronos and the Kronia“, „Saturnus and the Saturnalia" und „The Roman Festival for Bona Dea and the Greek Thesmophoria“). Richtungsweisend sind vor allem Burkert 1966 und Burkert 1972, 153-235 („Auflösung und Neujahrsfest") und 236-273 (,Anthesteria“). 
Auf der Inhaltsebene paßt in dieses Schema der immer wieder betonte $\mathrm{Ge}$ gensatz zwischen Alt und Neu (Jung), der in fast allen Stücken eine hervorgehobene Rolle spielt. Die Pole von Alt und Neu lassen sich auf der rituellen Referenzebene auf den Kampf zwischen Göttergeschlechtern, bzw. auf die Antithese von einer fernen Vergangenheit vor der Zivilisation und der Jetztzeit beziehen. Auch die Verjüngung von Alten spielt wie im Falle des Demos in den Rittern und des Philokleon in den Wespen eine Rolle. Der Aufbruch in eine phantastische Handlung hat meist utopische Züge. Die Utopie zeichnet sich, ähnlich wie in den genannten Festen die Übergangsperiode, durch eine extreme Ambivalenz zwischen einer Eu- und Dystopie aus. ${ }^{26}$

Besonders deutlich ist in den Komödien das Fest der Anthesterien präsent. Explizite Anspielungen darauf kommen in den Acharnern (Bruch mit der öffentlichen Ordnung in einer Polis, Schaffung eines Privatraumes, der durch den Ritus der Ländlichen Dionysien geheiligt wird, zuletzt Feier der Choes [Ach.

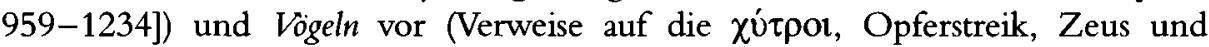
die Olympier werden zum Abdanken gezwungen, Heilige Hochzeit mit der Basilinna). ${ }^{27}$ Die Grundstruktur findet sich ebenso im Plutos (Opferstreik, Absetzung des Zeus und Installation eines neuen utopischen Gottes, Prozession mit $\chi \cup$ โ $\rho \circ)^{28}$ und im Frieden (Rückzug der olympischen Götter und Installation [Hidrysis] der Eirene, Heilige Hochzeit). ${ }^{29}$ Ein ähnliches Handlungsmuster offenbart sich auch in den Rittern (Sukzessionsmythos, Agon der alten mit einer neuen Ordnung, die den herkömmlichen Rahmen sprengt, Jungkochen des Demos und wundersame Rückkehr zum Alten), Wolken (Absetzung des Zeus, Auseinandersetzung zwischen alter und neuer Bildung und Religion), Wespen (der Konflikt zwischen Vater und Sohn entspricht dem Kampf von Alt mit Jung und wird zusätzlich mit Allusionen auf die Göttergenerationen untermalt) und in den Fröschen (der Agon zwischen dem alten Dichter Aischylos und dem neuen Dichter Euripides wird mit Anspielungen aus dem Kontext des Götterkampfs verstärkt; Dionysos steigt in seinem Heiligtum ह̉v $\lambda i ́ \mu v \alpha \iota \varsigma$ [216f.; vgl. 209-219] in die Unterwelt und holt die alte, chaotische Macht Aischylos zurück auf die Erde).

Solche Ausnahmefeste lassen sich gut auf dem Hintergrund der drei wichtigen Paradigmen der antiken Religionswissenschaft veranschaulichen. Dies sind namentlich die Initiation oder der rite de passage, das Neujahrsfest sowie die Vegetation und Fruchtbarkeit im Jahreszyklus. Nach Riten der séparation, mit Hilfe derer sich die Beteiligten einer Initiation symbolisch vom früheren Zustand

26 Zur Terminologie vgl. Auffarth 1991, 342 und Farioli 2001. Zur Utopie bei Aristophanes vgl. Schwinge 1977; Auger 1979; Zimmermann 1983; Farioli 2001.

27 Vgl. Craik 1987 und Auffarth 1994.

28 Vgl. Bierl 1994.

29 Vgl. Auffarth 1991, 546-549 und Bowie 1993, 146-150. 
verabschieden, gelangen sie nach van Genneps Schema in eine transitorische Phase der Liminalität. ${ }^{30}$ Gerade die Jugendlichen an der Schwelle zum Erwachsenendasein, beispielsweise auch die attischen Epheben, durchleben dabei einen antinormativen Zustand des ,Betwixt and Between'. In diesem kollabieren für sie alle Kategorien, vor allem primordiale Wildheit und neue Zivilisationsformen, bevor zuletzt nach der mit rituellen Praktiken markierten Statusänderung die Wiedereingliederung in die Gesellschaft erfolgt. ${ }^{31}$ Auch im Neujahrsfest stehen sich das Alte und das Neue in einem krisenhaften Übergang gegenüber, der ebenso wie das Pharmakosritual mit bäuerlichem Brauchtum und Vegetationskulten erklärt werden kann. ${ }^{32}$

Die drei Paradigmen treffen im Polisdiskurs zusammen. Biologische, jahreszeitliche und ,politische' Faktoren werden im athenischen Festkalender miteinander in eine analoge Beziehung gesetzt. Denn in den mythisch-rituellen Themen der biologischen Reifung und des Wechsels der Jahreszeiten gelingt es, den gerade für die Gesellschaft relevanten Ablauf vom Chaos zur Ordnung zu problematisieren. Dionysos, der Gott der Komödie, und die an seinen Festen virulenten Verkehrungstendenzen geben den gemeinsamen Nenner für die gattungsspezifischen Kennzeichen: ${ }^{33}$

Verkehrte Welt, Komödie und Dionysos

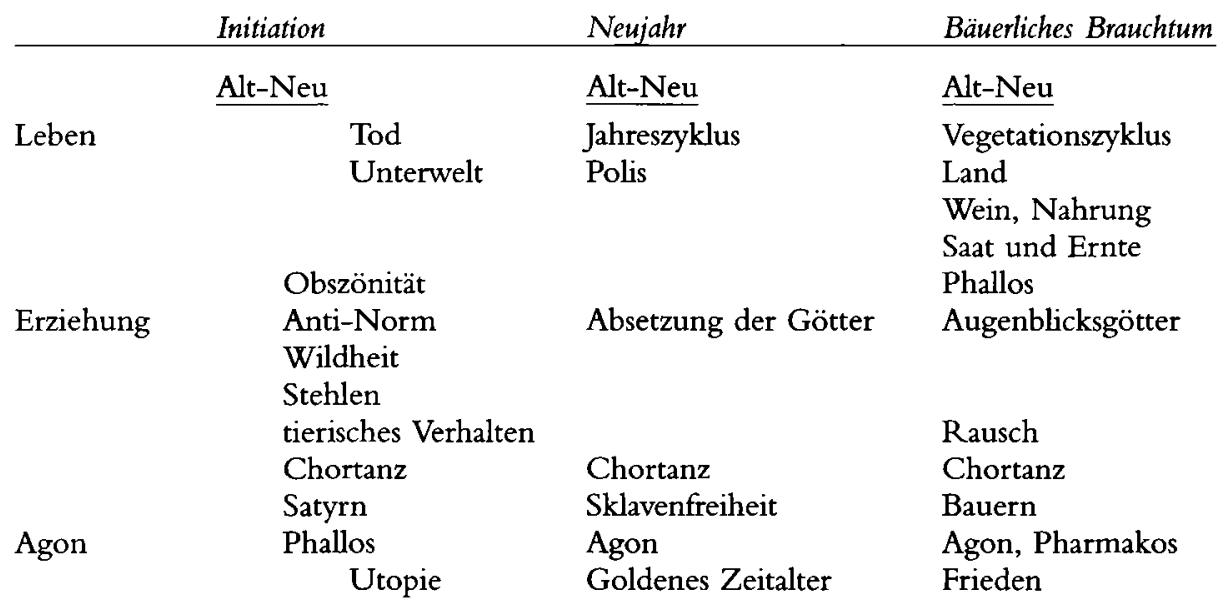

30 Vgl. van Gennep 1909.

${ }^{31}$ Zum Initiationskontext des komischen Chores vgl. Bierl 2001.

32 Vgl. auch Cornford 1914, bes. 66-77.

${ }^{33}$ Vgl. Hoffman 1989; allgemein zu Dionysos als Gott der coincidentia oppositorum vgl. Bierl 1991, 13-20. 


\section{Alt und Neu am Beispiel der Wolken}

Das Alte wird bei Aristophanes stets idealisiert, das Neue hat meist negative Konnotationen. Dabei wird die ferne Vergangenheit politisch auf die Marathonzeit übertragen, die bereits utopische Züge erhält (vgl. auch Nub. 986). Die Opposition kann schließlich einfach auf Generationenkonflikte, die typische Vater-Sohn-Thematik, appliziert werden. Die tempi passati werden in der Alten Komödie grundsätzlich verklärt: dort herrschen ländlich-bäuerliche Werte und Sitten sowie die dionysischen Merkmale der Komödie von ,Wein, Weib und Gesang. ${ }^{34}$ Vor allem gibt es im alten Zustand Sittlichkeit und Frieden, während das moderne Polisleben von Krieg, Entartung und Dekadenz bestimmt ist. Allerdings wäre es völlig verfehlt, diese Charakterisierung als Botschaft des Autors mißzuverstehen. Die Alte Komödie ist weder Ausdruck eines konservativen Traditionalismus noch vertritt Aristophanes eine pazifistische Grundhaltung. Die Aristophanische Komödie stellt als Kunstprodukt im Rahmen der dionysischen Okkasion keine Fortsetzung des Diskurses der Polis dar, sondern sie steht quer zu ihr. In einer raum-zeitlichen Enklave der Ausnahme spiegelt sich die immer neu hergestellte Anderwelt in der aktuellen Welt Athens, ohne mit ihr identisch zu sein. ${ }^{35}$ Beide Sphären beleuchten sich gegenseitig und stehen im Verhältnis der Komplementarität zueinander.

Eine tatsächliche Innovation liegt bei Aristophanes darin, wie er das strukturelle Schema auf diverse Weise umsetzt. Er bleibt nicht nur bei traditionellen Formen mit Hilfe von Chören, die in der Verkörperung von Tieren, Sklaven, Frauen, Barbaren, Unterweltsbewohnern etc. das Ambiente der grotesken Alterität für den Stoff eines Spieles schaffen. ${ }^{36}$ Wirklich neu und genial ist die Übertragung auf kulturelle Fragestellungen.

Wie oben geschildert, hat man im fünften Jahrhundert v. Chr. erstaunliche Innovationen auf dem Gebiet der Kultur, Dichtung, Wissenschaft, Philosophie und Rhetorik erzielt. ${ }^{37}$ Gerade die aufblühende Sophistik stellt das neue Paradigma schlechthin dar. Durch das Mittel der Rede kann man nach Protagoras ,das schwächere Argument zum stärkeren machen". 38 Sokrates und seine Denkerei (Phrontisterion) werden in den Wolken mit sämtlichen geistigen Strömungen der Zeit vermengt. ${ }^{39}$ Naturwissenschaftliche Spekulation und Theorie im Fahrwasser

$34 \mathrm{Vgl}$. Bierl 2001, 345.

35 Vgl. Riu 1999, 11-48, insbesondere zum Traditionalismus ebd. 41-46.

36 Vgl. Bierl 2001, 96-100.

37 Zur Analyse von Alt und Neu in den Wolken vgl. auch Marianetti 1992, 7-40. Vgl. Reckford 1991, 388-402, der die Schwellensituation der Wolken zwischen traditioneller und innovativ-origineller Komödie thematisiert.

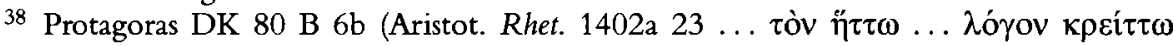

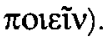

39 Vgl. MacDowell 1995, 113-149 als Einfuihrung. 
der Vorsokratik, religiöse Betrachtung und das Aufkommen des Atheismus sowie die Sophistik werden mitsamt orphisch-pythagoreischen Vorstellungen auf die historische Figur des Sokrates in komischer Verzerrung projiziert. ${ }^{40}$ Dieses phantastische Konstrukt Sokrates steht unter dem Schutz der Wolken, die den Chor bilden und die linguistische Semiose, also den permanenten Signifikationsvorgang, insbesondere die Umsetzung in sprachliche Zeichen, verkörpern. ${ }^{41}$ Das geistig Neue scheint das Mythisch-Alte vollends zu verdrängen. Ein Paradigma wird in der historischen Schwellenzeit des Aristophanes von einem anderen abgelöst.

Aus der komplementären Perspektive macht die Komödie anschaulich, wie beide Diskurse paradoxerweise miteinander verquickt sind, das Neue also auch vom Alten durchdrungen ist. Das Vorzeitige und das Aktuelle, das Einst und Jetzt sind auf komische Art, wie sich herausstellen wird, vollkommen verwirrt und verzerrt. Gleichzeitig bedingen sich das Mythisch-Archaische und das Wissenschaftlich-Topaktuelle gegenseitig. ${ }^{42}$

Diese Konstellation ist bereits in den Figuren angelegt. Strepsiades ist der Vertreter der Komödie, Verkörperung des traditionellen Ländlich-Bäurischen und des einfachen Lebens. Aufgrund der Verschwendungssucht und Pferdeliebhaberei seines mit einer Aristokratin gezeugten Sohnes Pheidippides, der bereits im Namen die Hybridität von sparsamem Bauerndasein und luxuriösem, dem Statussymbol des Pferdes sich verschriebenem Adel trägt, hat er immense Schulden angehäuft. Derer will er sich als Wort-,Verdreher' und mit anderen Tricksereien entledigen. Seine komische Idee liegt darin, den Sohn in die benachbarte Schule des Sokrates zu schicken, damit er dort die neue Kunst der eristischen Rhetorik erlernt, um die Gläubiger abzuschütteln. Pheidippides weigert sich freilich. Trotz seiner Jugend vertritt er zunächst die Position des auf der Zeusreligion fußenden Alten, der Dezenz, der Aristokratie, Sitte und Zucht, während der alte Vater schließlich aus seiner Not selbst zum Neuen getrieben wird. Er begibt sich nämlich selbst in die Schule. Der Übergang dorthin erweist sich als der notwendige komische Sturz in eine Anderwelt. Die Schüler treten als absurde, ausgezerrtbleiche Gestalten auf, die den sonst an Ausnahmefesten herumschwirrenden Totengeistern ähneln. Sie wie auch ihr Meister gehen völlig eigenartigen Beschäftigungen nach, wie zum Beispiel einer komisch-grotesken Geometrie und Astronomie sowie der Untersuchung der Dinge unter der Erde im Tartaros. Sie treiben eine basale Naturkunde und untersuchen dabei Fragen, wie weit etwa

40 Vgl. Marianetti 1992, 41-75, 108-132 und Patzer 1993. Zum Sokratesbild vgl. auch Schmid 1948; Erbse 1954; Gelzer 1956; Strauss 1966; Nussbaum 1980; Edmunds 1986; Edmunds 1987.

41 Vgl. Ambrosino 1983; zum Wolkenchor vgl. auch Segal 1969; Köhnken 1980; Gaertner 1999.

42 Vgl. Marianetti 1992, 7-40. 
der Floh springt oder ob Schnaken durch das Mundstück oder den Bürzel singen. Die Lösungen entsprechen dabei der Logik der Gattung.

Der Sukzessionsmythos von Uranos zu Kronos und von Kronos zu Zeus ist hier auf kunstvolle Weise mit dem allgemeinen Thema der verkehrten Welt verwoben. Im Hintergrund dieser Verkehrung der sinnstiftenden Normen stehen im Festkalender verankerte Kulte, in denen kurzzeitig die bestehende Ordnung außer Kraft gesetzt wird. In Festen wie den Kronia, Skira oder den Anthesterien werden, wie erwähnt, dabei in gleicher Weise die Götter kurzfristig abgelöst und der drohende Untergang der Welt bzw. utopische Alternativen durchgespielt, um schließlich zum Bestehenden als dem denkbar Besten zurückzukehren. Die Götter sind dementsprechend in den Wolken entmachtet und durch neue Gottheiten, die Wolken, das Chaos und den Wirbel sowie die Zunge (424), ersetzt. ${ }^{43}$

Gleichzeitig finden sich auch hier wieder Züge einer Initiation. ${ }^{44}$ Die verkehrte Welt ${ }^{45}$ und die Initiation treffen sich in der Übergangsphase des rite de passage der Einweihung, in dem für den Initianden ebenso wie den Epheben alle Werte und Normen auf den Kopf gestellt sind. Strepsiades unterzieht sich einer Initiation in die Schule des Sokrates, ${ }^{46}$ während dieser die Rolle eines komischen Doktors und diebischen Einweihungspriesters übernimmt. ${ }^{47}$ Für ihn ist jemand wie Strepsiades, der noch an Zeus glaubt, ein ,antediluvianischer Kauz, der nach

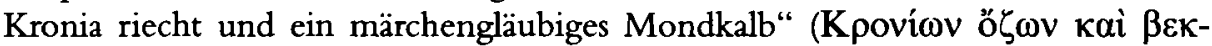

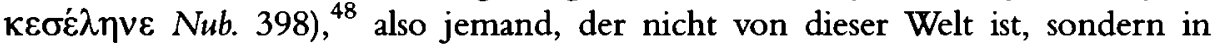
der grauen Vorzeit lebt.

${ }^{43}$ Die ,Dreifaltigkeit' ist genauso wenig stabil wie die Wolken; vgl. Nub. 264f. und 627.

44 Vgl. Bowie 1993, 102-112. Allerdings ist seine Anwendung des Paradigmas im Sinne einer Verkehrung problematisch.

45 Vgl. Kenner 1970; Versnel 1999; Farioli 2001.

46 Vgl. u. a. Dieterich 1893; Byl 1980; Marianetti 1992, 41-75; Marianetti 1993.

47 Vgl. Bowie 1993, 112-124. Diebstahl (Nub. 175-179, 497, 856, 1498) ist nicht nur Folge der Armut (so Patzer 1993, 86), sondern auch Zeichen des komischen Normbruchs. Vgl. auch Meynersen 1993.

$48 \mathrm{Vgl}$. Dover 1968, 152 ad loc., der das Element $\beta \varepsilon \kappa$ in $\beta \varepsilon \kappa \kappa \varepsilon \sigma \varepsilon \dot{\varepsilon} \eta v \varepsilon$ mit der berühmten Kaspar-Hauser-Geschichte bei Hdt. 2, 2 zusammenbringt. Psammetich ordnete ein Experiment an, um herauszufinden, welches Volk das älteste sei, Ägypten oder Phrygien. Das erste Wort der beiden von der Umwelt isolierten Kinder für Nahrung sei ßékoৎ gewesen, das phrygische Wort für ,Brot‘. Im Wort wie in der Komödie ist also Uraltes mit Barbarischem vermengt. Zum zweiten Element ,Mond" vgl. ebenfalls Dover 1968, 152 ad loc.; außerdem werden Sokrates und die Seinen als Leute gekennzeichnet, die den Sitz des Mondes auskundschaften (Nub. 1507). Zum astronomischen Interesse vgl. auch Nub. 172. Schießlich kann man die Thematik des Neumonds und des alten Mondkalenders damit assoziieren. Kronos nennt man auch einen altfränkischen Menschen; vgl. Nub. 929 und Vesp. 1480; vgl. auch Plut. 581. Zu Kronos und den Kronia vgl. Versnel 1993, 89-135. 
Nachdem aber Strepsiades am sokratischen Unterricht gescheitert ist, versucht der Vater seinen Sohn erneut zu überreden, für ihn die Schule zu besuchen. Als er sich weigert, wirft ihm sein Vater ,archaisches Denken“ vor (Nub. 821). Das Vordergründige hat er sich zu Herzen genommen: Zeus ist gestürzt, also soll man nicht mehr bei ihm schwören (Nub. 814ff.). Dinos, der Wirbel, hat jetzt die Herrschaft inne, nachdem er Zeus verjagt hat $(N u b .828$ in Aufnahme von $380 \mathrm{ff}$.), wobei die sprachliche Form eine Neuschöpfung nach dem ebenfalls ,goetischen' Empedokles darstellt. ${ }^{49}$ Allein die Ausdrucksweise markiert auf treffliche Weise die Spannung von Alt und $\mathrm{Neu}^{50}$

In Pheidippides' Augen ist sein Vater verrückt geworden (Nub. 846). Die grammatikalische Belehrung erachtet der Sohn als groben Unfug. Er nennt Sokrates und die Seinen $\gamma \eta \gamma \eta v \varepsilon \tilde{i} \varsigma$ (Nub. 853), das heißt Erdgeborene, die wie die grotesk-riesenhaften Giganten oder die Titanen den Zeushimmel stürzen wollen. Das Uralte bedroht in der Form des sokratisch-sophistischen Neuen offenbar die bestehende Ordnung.

Ein ähnlicher Schlagabtausch zwischen Alt und Jung wird in der Auseinandersetzung zwischen dem Logos Dikaios und Adikos fortgesetzt, zwischen denen sich Pheidippides nun zu entscheiden hat. Der Logos Adikos, der behauptet, Dike existiere nicht, da Zeus wegen des Sturzes seines Vaters Kronos nicht belangt worden sei (Nub. 904f.), bringt als Vertreter der sophistischen Moderne und neumodischen Erkenntnisse ( $\gamma v \tilde{\omega} \mu \alpha l$ kalvaí Nub. 896) das gegnerische Argument der Vergangenheit polemisch mit den archaischen Urgewalten in Verbindung:

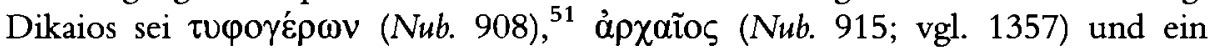
Kronos (Nub. 929, vgl. 1070).

Der Konflikt zwischen Vergangenheit und Moderne wird ebenso mit dem Sukzessionsmythos assoziiert. So meint der Logos Dikaios, daß, wer ihm folge, nie seinen Vater Iapetos - so heißt der aufrührerische Bruder des Kronos schelten oder ihn gar schlagen würde (Nub. 998f.). Umgekehrt herrschte zur Zeit des Kronos bekanntlich das Goldene Zeitalter. Daher ist der Preis der guten alten Zeit durch den Logos Dikaios ebenso mit den Zeichen der Kronosherrschaft kodiert. Die grundsätzliche Dialektik der Kronosepoche ist in den dissoi logoi der Sophistik aufgehoben, die Sokrates mit seiner Schule unter anderem auch verkörpert.

Gerade der folgende Wortwechsel der beiden Logoi macht den Gegensatz zwischen der Vorzeit und einer dynamischen Aktualität deutlich (Nub. 984-986):

49 Vgl. Willi 2003, 101-105 und 110. Zum empedokleischen Hintergrund des Sokrates ebd. 110-113. Zu Empedokles als goes vgl. Bierl (in Vorbereitung).

${ }^{50} \mathrm{Zu}$ den linguistischen Neuerungen vgl. Noël 1997 und Willi 2003, 96-156.

51 Auch die Vertreter des Neuen Dithyrambos singen von Typhon (Nub. 336). 


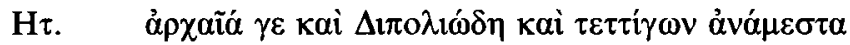

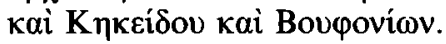

K $\rho$.

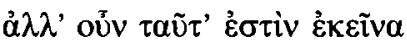

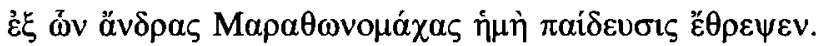

Schwächerer L.: Altvätrisches Zeug, altfränkischer Brauch der Dipolieia, voll von Zikaden, verklungener Musik eines Keikeides und Buphonien!

Stärkerer L.: Ja freilich! Doch war dies genau jene Zeit, wo erzogen durch mich das Heroengeschlecht der Marathonkämpfer

heranwuchs. ${ }^{52}$

Hier zeigt sich erneut, wie der Diskurs der Gerechtigkeit mit der Urzeit, mit den Kronia und anderen uralten Bräuchen und Riten verbunden ist, ${ }^{53}$ die für die Polis von enormer Wichtigkeit und längst nicht passé sind. Zum anderen wird deutlich, wie die Marathon-Epoche als Goldene Zeit utopische Züge annimmt.

Schließlich kommt der Sohn aus der Schule und schlägt in einer Auseinandersetzung, welcher Dichter besser sei, der archaische Simonides und Aischylos oder der moderne Euripides, als neuer "Zeus“ seinen Vater „Kronos" (vgl. Nub. 904f.). Pheidippides vermag dabei noch dazu in sophistischer Manier zu beweisen, daß es zu Recht geschieht (Nub. 1391-1477).

Nach dem zweiten Agon ist Strepsiades also endgültig gescheitert. Er ist wieder der Alte und kehrt, wenn auch noch immer grotesk verzerrt, zur Zeusordnung und -religion zurück. Er befrägt Hermes und zerstört das Phrontisterion, indem er es mit einer Leiter besteigt und von oben in Brand setzt. ${ }^{54}$ Der Sohn Pheidippides ist dagegen ab seiner Initiation mit dem Unwesen des Neuen assoziiert.

Eng mit dem Gegensatz von Alt und Neu ist in den Wolken die für die Sophistik so entscheidende Opposition von Nomos und Physis verbunden und ko-

52 Das Fest namens Dipolieia oder Buphonia wurde zur Erinnerung an die erste Begründung des Ackerbaues gegen Ende Juni (14. Skirophorion) für Zeus Polieus mit besonders archaischen Riten gefeiert. Vgl. Burkert 1972, 153-161.

${ }^{53} \mathrm{Vgl}$. Nub. 915 und 929. Die Zikaden galten selbst als Erdgeborene ( $\gamma \eta \gamma \varepsilon v \varepsilon i \tilde{\zeta}$ [vgl. Nub. 853]) und Verwandte der Urmenschen; vgl. Nub. 984f. mit Scholien. Daher steckte man sich am Buphonienfest goldene Zikaden ins Haar, nachdem man an den Skira an die Erdgeburt des Erichthonios erinnert hatte. Zur Zikade als Tier und Symbol der Goldenen Zeit vgl. Baudy 1992, 20. Vgl. auch Nub. 1360. Offenbar verkleideten sich Epheben als Zikaden und stellten initiatorische Tierchöre dar. Zu den Skira und der Pyrrhiche vgl. Bierl 2001, 233-251, bes. 236-239. Daher wird der Waffentanz an den folgenden Panathenäen auch so sehr vom Logos Dikaios thematisiert; vgl. Nub. 961-989.

54 Auffarth 1999, 93-96 sieht das Ende als Wiederaufnahme der Initiationshandlung. Durch die Feuerfackel werde die richtige Erkenntnis des Strepsiades symbolisiert. Wahrscheinlich wird er eher zum komischen Stürmer des neuen Himmels. 
misch dekonstruiert. ${ }^{55}$ Die überlieferte Konvention wird in der Sophistik bekanntlich durch das Naturrecht aufgebrochen, der alte Nomos also durch die Rhetorik, moderne Philosophie und atheistische Theoreme zerstört.

Sokrates und seine Denkerei stehen hier eher auf der Seite der Physis, die von der Sophistik in den Vordergrund gerückt wird. Als Erklärung für die Phänomene der Realität wird bevorzugt die Natur hervorgehoben. Trotzdem geht es Sokrates und den Wolken darum, die Doppelbödigkeit der Welt und ihre Begründung sowohl auf Nomos als auch Physis aufzuzeigen.

In der Unterweisung in Metrik, Rhythmik und Grammatik behandelt Sokrates die Konventionen der Sprache (Nub. 627-699). ${ }^{56}$ Der Logos entzweit das kohärente System, hinterfrägt es und macht die Unstimmigkeiten der Normen deutlich. Durch eine komische Analyse wird somit das Chaos hinter der Ordnung sichtbar. Mit Hilfe der Sprache finden also entsprechend vorsokratischen Vorstellungen Hybridität und Verwirrung erst Eingang in die Welt. Der Wolkenchor referiert in der ersten Parabase die Klage des Mondes über die Unordnung des Kalenders (Nub. 615-626) - durch die aus dem altattischen System resultierende Differenz der tatsächlichen mit den kalendarischen Neumonden verschoben sich auch die Feste - und bestätigt auf komische Weise die Verzerrung im Stück. Die Wolken sind wie die Komödie insgesamt polyvalent. Zum einen haben sie in der Perspektive der Physis ein tauiges, irdisches und urtümliches Wesen (Nub. 275-290, 298, 330-338), zum anderen werden sie als hochfliegend, von der irdischen Haftung enthoben und als Ernährer der hochtrabenden Dithyrambendichter und Sophisten (Nub. 331-334) beschrieben und gelten dadurch als Vertreter der Moderne. Ferner sind sie entweder einfach Gebilde aus Wasser, Dampf und Nebel, phantastische Luftschlösser oder göttliche Wesen.

In beiden Parabasen weist der Wolkenchor auf seine zweischneidige Kraft hin: Die Wolken stehen einerseits im Dienst einer zivilisationsstiftenden Gesetzmäßigkeit der Natur, indem sie einen geregelten Ackerbau garantieren. Daher fordern sie in der Hauptparabase ihren Kult gemäß den konventionalisierten Riten der attischen Religion ein. Als höhere Instanz sind sie wie Zeus Teil des tradierten Kosmos. Sie verkörpern die gerechte Satzung der Dike, die ein Leben in der Polisgemeinschaft ermöglicht. Die Wolken können andererseits als ungestüme Naturkräfte diese Ordnung ebenso zerstören, falls die Menschen nicht an die Götter glauben. Sie sind nämlich in der Lage, die Felder verdorren zu lassen und Unfruchtbarkeit zu bringen. Ferner verfügen sie über die Macht, die Häuser der Menschen durch Hagel zu verwüsten sowie die Verbindung von Frau und Mann durch Regen zu verhindern (Nub. 1115-1130). Schließlich können sie sich sogar

55 Vgl. Heinimann 1945, 131; Nussbaum 1980, 52-63; Hubbard 1991, 112.

56 Vgl. Willi 2003, 98-100. 
in die Politik der Polis einmischen, wie im Epirrhema der Parabase (Nub. 575-594) gezeigt wird.

Die Wolken und Sokrates verführen Strepsiades auf den reinen Weg der Physis, obwohl die Notwendigkeit des Korrektivs durch den Nomos deutlich wird. Die neue, von Sokrates und den Wolken propagierte Religion basiert ausschließlich auf der Natur. Ich stelle die Zusammenhänge zunächst graphisch dar:

Nomos

Physis

Zeus

naturwiss. Forschung

Physiologie der Vorsokratiker

trad. Theologie

sophistischer Atheismus

neue Religion

myth. Vorstadium

Orphik, Hesiod

Chaos, Dinos, Aither etc.

Aristophanes' Komik liegt also darin, den Physis-Begriff in zwei Bereiche aufzufächern. In der Abfolge von Nomos/herkömmlicher Theologie zu Physis/naturwissenschaftlich-sophistischem Atheismus wird einerseits ein zeitliches Kontinuum im Sinne eines Fortschritts von Alt zu Neu evoziert. Andererseits implizieren die mit der Physis verbundenen Begriffe auch ein zirkuläres Zurück in eine mythische Urzeit vor der Konsolidierung der Zeusherrschaft. Chaos ist das leere Urgähnen, aus dem sich nach Hesiods Theogonie (116ff.) der Kosmos entwickelte. Zuerst war Chaos, wenig später Gaia. Sie bilden den Anfang. Dann kommen Tartaros und Eros hinzu. Aus Chaos entstehen Erebos und Nyx. Nyx gebiert Aither; Gaia bringt Uranos, die Berge und Pontos hervor. Mit Uranos zeugt sie „Okeanos, den tiefen voller Wirbel“ (' $\Omega \kappa \varepsilon \alpha v o ̀ v ~ \beta \alpha \theta v \delta i ́ v \eta v$ Hes. Th. 133) und die Titanen, unter anderen Iapetos und Hyperion, zudem die Kyklopen, Brontes und Steropes zuerst, die Donner und Blitz für Zeus erschaffen. Uranos ist bekanntermaßen der Vater des Kronos. Aus der Vereinigung von Kronos mit Rhea entsteht Zeus.

In dieser Vorzeit sind also das primäre Chaos (Nub. 424, 627) sowie Nebel, Wolken, Aither, Luft, Atem und Wirbel anzusetzen, die wiederholt als neue göttliche Instanzen angesprochen werden ( $N u b$. 264f., 424, 627, 814). Göttergeschlechter lösen sich bekanntlich gewaltsam ab. Ich erinnere an die Titanenund Gigantenkämpfe. Der Glaube an die neuen Götter ist also in Wahrheit ein temporäres Zurück in eine bereits überwundene Entwicklungsstufe der Menschheit und des Kosmos. Er versteht sich also nicht als Atheismus, sondern als die wiedererwachte Verehrung der Urmächte der Natur, die im Laufe der Geschichte längst unter die neue Zeusreligion subsumiert worden waren.

Die Natur ist voller Ambivalenz. Einerseits stellt sie eine urtümliche Gewalt dar, welche die Zivilisation sprengen kann, andererseits wird sie für die Entwicklung des Ackerbaus und jeglicher Kultur gebraucht. Daher steht sie ebenso unter der Herrschaft des Zeus. Die Wolken sind Teil der doppelschneidigen Physis, die im Nomos der Zeusherrschaft integriert und aufgehoben werden muß. Falls jedoch die Einbettung in den Nomos entfällt, bricht die Macht der Wolken und 
der Natur ungezügelt hervor und bedroht die Welt mit Chaos, verkehrter Welt und Untergang.

Somit erklären sich sowohl die traditionellen Oden des Wolkenchors auf die olympischen Götter als auch seine sogenannte ,Wende' am Ende. Die Wolken stehen nämlich in Wahrheit nicht in Opposition zu Zeus, sondern sie sind Teil seiner Herrschaft über den gesamten Kosmos. Daher ist Zeus als Wettergott im

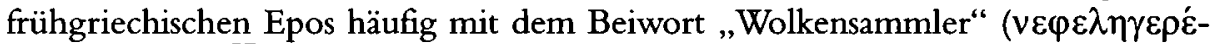
$\tau \alpha)$ verbunden. ${ }^{57}$

Die Wolken führen Strepsiades an der Nase herum (Nub. 344ff.) und machen ihm zusammen mit ihrem Weihepriester Sokrates weis, daß es Zeus nicht mehr gibt. Strepsiades lernt in seiner Beschränktheit nicht die dissoi logoi, sondern die komplexe Lehre wird ausschließlich auf das Prellen, Betrügen und Überlisten reduziert. Die Komik liegt darin, daß der bäurische ,Naturmensch' Strepsiades ausschließlich die Argumente der Natur rezipiert. Als er von Sokrates gefragt wird, wie er sich der Gläubiger entledigen könnte, rezitiert er nur ein komisches Konzentrat der eben erlernten Naturphilosophie. Er schlägt ein Einsperren des Mondes, ein Einschmelzen der Wachsanklageschrift durch Feuer aus dem Brennglas oder die Ausflucht durch Selbstmord vor (Nub. 749-782). Schließlich vertreibt er die Schuldeneintreiber mit einer komischen Wiederaufnahme der sokratischen Argumente und mit Brachialgewalt (Nub. 1214-1302). Seine Begründung auf dem natürlichen Recht des Stärkeren bedroht allerdings das Zusammenleben der Bürger unter den festen Regeln der Dike.

Pheidippides wird erst im Laufe der Handlung zum Instrument der Wolken. Durch sein Eingreifen wird die Hierarchie zwischen Alt und Jung, Vater und Sohn außer Kraft gesetzt. Strepsiades' Strafe besteht darin, von seinem Sohn konsequent den Spiegel vorgehalten zu bekommen. Er schlägt ihn, wie Strepsiades die Gläubiger schlug. Als dieser mit der Vergeltung des Zeus droht, entgegnet ihm Pheidippides mit den Sophisma, daß der Herrscher des Olymps durch

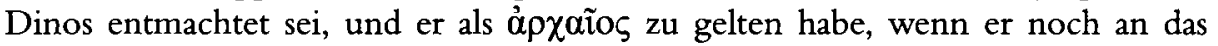
alte Zeug der Zeusreligion glaube (Nub. 1469-1471).

Das Ende (Nub. 1478-1511) bleibt offen. Entweder wollen die Götter wirklich die Bestrafung des Sokrates - Strepsiades wäre somit ausführendes Organ

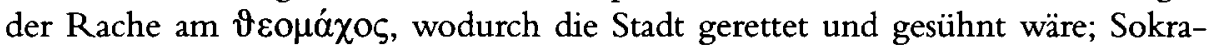
tes und Pheidippides dienen dabei als Modelle des Pharmakos. ${ }^{58}$ Nach dem Chaos wäre dadurch die Ordnung wiederhergestellt. ${ }^{59}$ Oder, die wahrscheinlichere Alternative: Strepsiades projiziert sein Handeln auf die Götter, die ihn weiterhin verspotten. Die Orgie der Gewalt gegen das Phrontisterion paßt auch zu

57 Z. B. Hom. Il. 1, 511; es gibt 47 Belege im frühgriechischen Epos.

58 Vgl. Nub. 1449.

59 Nach Segal 1969, 195 ist die Aktion als Wiederherstellung des alten Strepsiades zu deuten. 
seinem Wesen. Er hat nichts gelernt und bleibt seiner ungehemmten Natur treu. Er hält sich nicht an die Dike und den Nomos Athens, nämlich mit legalen Mitteln einen Prozeß gegen Sokrates zu führen, sondern schreitet erneut zu Gewalt, mit Feuer und Hacke, ähnlich den ungestümen Giganten, die mit einer Leiter den Himmel stürmten.

Insgesamt wird also dem Publikum auf der Bühne ein komisches Ende der Zeusherrschaft und der Poliszivilisation inszeniert. Das Chaos bricht auf komische Weise in der Gestalt des Sokrates und des Initianden Strepsiades herein. Die Abschaffung des theologischen Systems wird vor der versammelten Stadt durchgespielt. Der wissenschaftlich-sophistische Fortschritt wird als Zurück in die graue Vergangenheit des Schöpfungsmythos karikiert und durch das gemeinschaftliche Lachen wird die verkehrte Welt nach Beendigung des Stücks wieder gebannt. Zeus und die Ordnung, die auf Kult und politischen Normen beruht, werden schließlich gestärkt. Denn das Abschaffen der Werte erzeugte letztlich keine gangbare Alternative. Umgekehrt konnte dem Publikum die Komplexität der modernen Theorien angedeutet werden, die sich als weitaus differenzierter erweisen, als im Stück vorgetragen wurde. Nicht die Philosophie selbst, sondern die naive Interpretation der Bürger stellt die eigentliche Gefahr für die Stabilität dar. Die verkehrte Welt, die im realen Athen ihren Spiegel hat, wird neben dem Beispiel der grammatischen Illogizität im Genusgebrauch bei Substantiven mit männlicher Endung und weiblichem Artikel auch anhand konventionalisierter

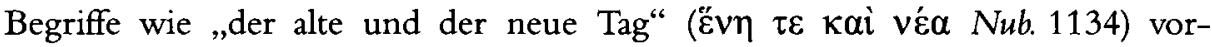
gestellt, der die Zweiheit in der Einheit, die krisenhafte Verschränkung der Zustände, versinnbildlicht. ${ }^{60}$ Er ist der Tag des Neumonds, also des Übergangs. An ihm werden auch die Schulden eingetrieben. Werden sie nicht gezahlt, wird der Nomos durchbrochen.

Die Anthesterien stellen, wie erwähnt, ein typisches Fest der verkehrten Welt dar. Hier wird die Ordnung ebenso vom kurzzeitigen Chaos bedroht. Man versuchte an solchen Tagen auch die Rechnungen zu begleichen. Athenaios (437d) berichtet vom Brauch, am Tage der Choen den Lehrern Geschenke und die

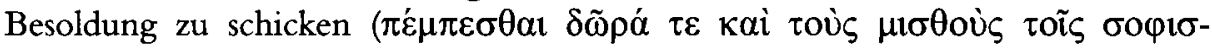
$\tau \alpha i ̃ \varsigma)$. Von dieser Notiz kann man einen ironischen Blick auf den Schluß des Stückes werfen. Strepsiades ist offenbar noch nicht geheilt. Er begleicht auf seine Weise die Rechnung mit den Sophisten, indem er das hereingebrochene Chaos mit einer ,wirbelnden' Gewaltorgie in einer Art Wüstung fortsetzt, ${ }^{61}$ die an die

60 Durch die Kalenderverschiebung zwischen Sonnen- und Mondjahr gab es ein strittiges Grenzgebiet für den bürgerlichen Monatsanfang. Vgl. den Sophismus, zwei Tage können nicht einer sein (Nub. 1178-1200 und 1222 f.). Vgl. Willi 2003, 98 und Walsh 1981.

61 Vgl. Meuli 1953, 234 und Davies 1990. 
Zerstörung des Versammlungshauses der Pythagoreer in Kroton im zweiten Viertel des fünften Jahrhunderts v. Chr. erinnert.

\section{Kurzer Ausblick auf die Vögel}

In den Vogeln haben wir eine ganz ähnliche Situation vor uns. ${ }^{62}$ Es geht erneut um die Abschaffung der Olympier durch die neuen Götter der Vögel, also um ein Weiterspinnen der Sukzessionsmythen im modischen Kontext der Sophistik und Rhetorik. ${ }^{63}$

Peisetairos' Reise ins Land der Vögel läßt sich ebenfalls als Weg in eine atavistische Anderwelt in komischer Vermengung mit modernem, sophistischem Gedankengut verstehen. Die geniale Idee der Gründung einer neuen utopischen Stadt namens Wolkenkuckucksheim eröffnet wiederum eine komplementäre Sicht auf das aktuelle Athen. ${ }^{64}$ Und erneut wurde die Komödie mit den Anthesterien und anderen orientalischen Mythen und Kulten des Sturzes der Götter in Verbindung gebracht. ${ }^{65}$ Die Einführung des Neuen bedeutet zugleich wie in den Wolken einen Rückfall in ein primordiales Zivilisationsniveau. Zugrunde liegen verschiedene Riten der Ausnahme und des Übergangs, in denen die temporäre Auflösung des Bestehenden und die Rückkehr zur Ordnung begangen werden. Dabei wird stets eine Urzeit und die Kulturentstehung als Schöpfungsgeschichte evoziert. ${ }^{66}$

Nicht von ungefähr steht in der Parabasen-Rede (Av. 685-722) des Peisetairos, in der der Machtanspruch der Vögel begründet wird, das Ursprüngliche im Zentrum. In der Komödie wird nämlich eine Zeit reaktualisiert, als Mensch, Tier und Gott noch nicht eindeutig voneinander geschieden waren. Im Mythos der Griechen wird diese Trennung erst durch Prometheus vollzogen. Daher spielen diese myhisch-rituellen Elemente eine herausragende Rolle im Plot. Die einzelnen Teile der Schöpfungsgeschichte werden vom Dichter aber in karnevalesker Freiheit zusammengesetzt. ${ }^{67}$ Das neue Element der Physis in der Tierwelt ist zugleich wiederum uralt. Um eine Herrschaft auf der Basis der Physis ohne Nomos zu errichten, wird das Neue mit dem Argument des Uralten abgesichert und legitimiert. Der Aufbruch in eine neue Alternative gerät schließlich zu einem Zurück in eine unheimliche Vergangenheit.

62 Vgl. MacDowell 1995, 199-228 als Einführung.

63 Vgl. Hofmann 1976, bes. 79-90 zur Folie der Gigantomachie, in welche die Titanomachie eingelegt ist. Zum Thema des neumodischen Atheismus vgl. Romer 1994.

64 Vgl. Bertelli 1983; Corsino 1987; Hubbard 1997. Zur Verarbeitung von Gründungsmythen vgl. Bowie 1993, 152-166.

65 Vgl. Craik 1987 und Auffarth 1994.

66 Vgl. Zannini Quirini 1987.

67 Vgl. Ricciardelli Apicella 1993 und West 1994. 
Wie so oft wird auch hier eine Welt eines anfänglichen Chaos und des ganz Anderen auf der attischen Bühne lebendig. Die ambivalenten Vögel übernehmen die Herrschaft, indem sie sich ihr altes Recht zurückholen. Als Herren des Luftraumes (Chaos) blockieren sie die olympischen Götter im Himmel und zwingen die Menschen auf Erden unter ihr Regime. Die Schöpfung wird nicht im Sinne einer Wiedergewinnung der jetzigen Lebensform durchgespielt, sondern nach dem Zurück zum Ursprung wird eine alternative orphische Kulturentstehung aufgebaut, die die Gründung der Vogelstadt untermalt. ${ }^{68}$ Das Alte installiert sich als Neues. Die Rückkehr zur Normalität muß vom Zuschauer alleine vollzogen werden. Auf der Bühne etabliert sich die neue Macht der uralten Vögel unter der Regie eines machthungrigen Atheners, der sich zum Tyrannen und verzerrten Abbild des olympischen Göttervaters aufschwingen konnte. Die Bestätigung des alten Kosmos geschieht aufgrund des Lachens über die Absurdität der grotesken neuen ,Zeus'-Ordnung.

\section{Schluß}

Es wäre ein lohnenswertes Unterfangen, diesen Ansatz auf alle Aristophanischen Komödien auszuweiten. Insgesamt konnte gezeigt werden, wie die Thematik des Alten und Neuen in der Gattung der Alten Komödie nicht nur, wie wohl kaum seitdem in anderen literarischen Werken, von herausragender Bedeutung, sondern sogar konstitutiv ist. Das in einem Sitz im Leben, der Okkasion der dionysischen Feste, verankerte Genre hat in einer weitgehend mündlich geprägten Kommunikationsform, in der Mythos und Ritual entscheidende Bezugsmakrotexte darstellen, die Funktion, in einer Reise in eine atavistisch-primordiale Anderwelt eine komplementäre Sicht auf die Gegenwart zu eröffnen. Hier hat sich vor allem das rituelle Muster von Ausnahmefesten als fruchtbarer Hintergrund erwiesen. Der geniale und originelle komische Einfall des Dichters liegt darin, dieses Schema immer wieder mit zeitgenössischen Themen auszufuillen. Dabei wird ebenso in der Plotgestaltung Neues in der Verschränkung mit Uraltem problematisiert. Die Konstruktion einer verkehrten Welt im Neuen auf der Folie des Archaischen schafft das notwendige Ridiculum. Aus der komisch-verzerrten Perpektive, die komplementär zur aktuellen Welt der Polis steht, schöpft der antike Zuschauer in der kollektiven Erfahrung des Lachens Kraft und Heilung, um den Strapazen und Frustrationen des Alltags gewachsen zu sein. 


\section{Literaturverzeichnis}

Ambrosino 1983

Auffarth 1991

Auffarth 1994

Auffarth 1999

Auger 1979

Baudy 1992

Bertelli 1983

Bierl 1991

Bierl 1994

Bierl 2001

Bierl 2002a

Bierl 2002b

Bierl (in Vorbereitung)

Bing 1988
D. Ambrosino, Nuages et sens. Autour des Nuées d'Aristophane, Quaderni di Storia 18, 1983, 3-60.

C. Auffarth, Der drohende Untergang. ,Schöpfung in Mythos und Ritual im Alten Orient und in Griechenland am Beispiel der Odyssee und des Ezechielbuches (Religionsgeschichtliche Versuche und Vorarbeiten 39), Berlin und New York 1991.

C. Auffarth 1994, Der Opferstreik: Ein altorientalisches ,Motiv bei Aristophanes und im homerischen Hymnus, Grazer Beiträge 20, 1994, 59-86.

C. Auffarth, Ein seltsamer Priester und seine Jünger. Typisches und Charakteristisches im Bühnen-Sokrates des Aristophanes, in: K. Pestalozzi (Hrsg.), Der fragende Sokrates (Colloquium Rauricum 6), Stuttgart und Leipzig 1999, 77-97.

D. Auger, Le théâtre d'Aristophane: Le mythe, l'utopie et les femmes, in: D. Auger, M. Rosellini und S. Said (Hrsg.), Aristophane: Les femmes et la cité (Les cahiers de Fontenay 17), Fontenay-aux-Roses 1979, 71-101.

G. J. Baudy, Der Heros in der Kiste. Der Erichthoniosmythos als Aition athenischer Erntefeste, Antike und Abendland 38, 1992, 1-47.

L. Bertelli, L'utopia sulla scena: Aristofane e la parodia della città, Civiltà classica e cristiana 4, 1983, 215-261.

A. F. H. Bierl, Dionysos und die griechische Tragödie. Politische und ,metatheatralische' Aspekte im Text (Classica Monacensia 1), Tübingen 1991.

A. Bierl, Karion, die Karer und der Plutos des Aristophanes als Inszenierung eines anthesterienartigen Ausnahmefestes, in: A. Bierl und P. von Möllendorff (hrsg. unter Mitwirkung von S. Vogt), Orchestra. Drama-Mythos-Bühne, Stuttgart und Leipzig 1994, 30-43.

A. Bierl, Der Chor in der Alten Komödie. Ritual und Performativität (Beiträge zur Altertumskunde 126), München und Leipzig 2001.

A. Bierl, ,Viel Spott, viel Ehr!" - Die Ambivalenz des ỏvo $\mu \alpha \sigma \tau i$ $\kappa \omega \mu \omega \delta \varepsilon \tilde{i} v$ im festlichen und generischen Kontext, in: A. Ercolani (Hrsg.), Spoudaiogeloion. Form und Funktion der Verspottung in der aristophanischen Komödie (Drama 11), Stuttgart und Weimar 2002, 169-187.

A. Bierl, Experimentelle Innovation und ihre rituell-pragmatischen Grenzen in der Alten Komödie, Quaderni Urbinati di Cultura Classica n.s. 72.3 (101), 2002, 7-21.

A. Bierl, Empedokles: Philosoph, Arzt, Dichter und religiöser Prophet. Vom Mythos zum Logos? (Basler Antrittsvorlesung, 13. 5. 2003, Publikation in Vorbereitung).

P. Bing, The Well-Read Muse. Present and Past in Callimachus and the Hellenistic Poets (Hypomnemata 90), Göttingen 1988. 
Bowie 1993

Brelich 1975

Burkert 1966

Burkert 1972

Buxton 1999

Byl 1980

Cornford 1914

Corsino 1987

Craik 1987

Davies 1990

Dieterich 1893

Dover 1968

Dover 1972

Edmunds 1986

Edmunds 1987

Erbse 1954

Farioli 2001

Gaertner 1999

Gelzer 1956

Grilli 2001
A. M. Bowie, Aristophanes. Myth, Ritual and Comedy, Cambridge 1993 .

A. Brelich, Aristofane: commedia e religione, in: $M$. Detienne (Hrsg.), Il mito. Guida storica e critica, Roma und Bari ${ }^{3} 1982$ (1975), 103-118.

W. Burkert, Kekropidensage und Arrhephoria. Vom Initiationsritus zum Panathenäenfest, Hermes 94, 1966, 1-25 (ND in: W. Burkert, Wilder Ursprung. Opferritual und Mythos bei den Griechen [Kleine Kulturwissenschaftliche Bibliothek 22], Berlin 1990, 40-59).

W. Burkert, Homo Necans. Interpretationen altgriechischer Opferriten und Mythen (Religionsgeschichtliche Versuche und Vorarbeiten 32), Berlin und New York ${ }^{1} 1972,{ }^{2} 1997$.

R. Buxton (Hrsg.), From Myth to Reason? Studies in the Development of Greek Thought, Oxford 1999.

S. Byl, Parodie d'une initiation dans les Nuées d'Aristophane, Revue Belge de Philologie et d'Histoire 58, 1980, 5-21.

F. M. Cornford, The Origin of Attic Comedy, Cambridge 1914, ${ }^{2} 1961$ (edited with foreword and additional notes by T. H. Gaster), repr. with introduction by J. Henderson, Ann Arbor 1993.

E. Corsino, Gli Uccelli di Aristofane: utopia o satira politica, in: R. Uglione (Hrsg.), La città ideale, Torino 1987, 57-136.

E. M. Craik, ,One for the Pot': Aristophanes' Birds and the Anthesteria, Eranos 85, 1987, 25-34.

M. Davies, ,Popular Justice“ and the End of Aristophanes' Clouds, Hermes 118, 1990, 237-242.

A. Dieterich, Über eine Szene der aristophanischen Wolken, Rheinisches Museum 48, 1893, 275-283 (zitiert nach ND in: Kleine Schriften, Leipzig und Berlin 1911, 117-124).

Aristophanes, Clouds, ed. with introd. and comm. by K. J. Dover, Oxford 1968, Paperback 1989.

K. J. Dover, Aristophanic Comedy, London, Berkeley und Los Angeles 1972.

L. Edmunds, Aristophanes' Socrates, Proceedings of the Boston Area Colloquium in Ancient Philosophy, Band 1, Lanham 1986, 209-230.

L. Edmunds, Il Socrate aristofaneo e l'ironia prattica, Quaderni Urbinati di Cultura Classica n.s. 26.2 (55), 1987, 7-21.

H. Erbse, Sokrates im Schatten der aristophanischen Wolken, Hermes 82, 1954, 385-420.

M. Farioli, Mundus alter. Utopie e distopie nella commedia greca antica, Milano 2001.

J. F. Gaertner, Der Wolken-Chor des Aristophanes, Rheinisches Museum 142, 1999, 272-279.

T. Gelzer, Aristophanes und sein Sokrates, Museum Helveticum 13, 1956, 65-93.

A. Grilli, Il poeta comico e i vincoli del repertorio. L'esempio di Aristofane, in: A. Grilli und A. Simon (Hrsg.), L'officina del 
Halliwell 1991

Heinimann 1945

Hoffman 1989

Hofmann 1976

Hubbard 1991

Hubbard 1997

Kenner 1970

Köhnken 1980

Lohr 1986

Lowe 1988

MacDowell 1988

MacDowell 1995

Manuwald 2000

Marianetti 1992

Marianetti 1993

Meier 1980

Meuli 1953

Meynersen 1993 teatro europeo, Band 1: Performance e teatro di parola, Pisa 2001, 13-28.

S. Halliwell, The Uses of Laughter in Greek Culture, Classical Quarterly 41, 1991, 279-296.

F. Heinimann, Nomos und Physis. Herkunft und Bedeutung einer Antithese im griechischen Denken des 5. Jahrhunderts, Basel 1945.

R. J. Hoffman, Ritual License and the Cult of Dionysus, Athenaeum n.s. 67, 1989, 91-115.

H. Hofmann, Mythos und Komödie. Untersuchungen zu den Vogeln des Aristophanes (Spudasmata 33), Hildesheim und New York 1976.

T. K. Hubbard, The Mask of Comedy. Aristophanes and the Intertextual Parabasis (Cornell Studies in Classical Philology 51), Ithaca und London 1991.

T. K. Hubbard, Utopianism and the Sophistic City in Aristophanes, in: G. W. Dobrov (Hrsg.), The City as Comedy. Society and Representation in Athenian Drama, Chapel Hill und London 1997, 23-50.

H. Kenner, Das Phänomen der verkehrten Welt in der griechisch-römischen Antike (Aus Forschung und Kunst 8), Klagenfurt 1970 .

A. Köhnken, Der Wolken-Chor des Aristophanes, Hermes 108, 1980, 154-169.

G. Lohr, Körpertext. Historische Semiotik der komischen Praxis, Opladen 1986.

N. J. Lowe, Greek Stagecraft and Aristophanes, in: J. Redmond (Hrsg.), Farce (Themes in Drama 10), Cambridge 1988, 33-52.

D. M. MacDowell, Clowning and Slapstick, in: J. Redmond (Hrsg.), Farce (Themes in Drama 10), Cambridge 1988, 1-13.

D. M. MacDowell, Aristophanes and Athens. An Introduction to the Plays, Oxford 1995.

B. Manuwald, Zur Dialektik von ,alt' und ,neu' in der griechischen Tragödie, Antike und Abendland 46, 2000, 76-92.

M. C. Marianetti, Religion and Politics in Aristophanes' Clouds (Altertumswissenschaftliche Texte und Studien 24), Hildesheim 1992.

M. C. Marianetti, Socratic Mystery-Parody and the Issue of

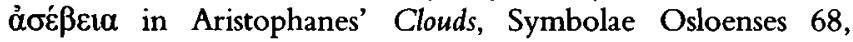
1993, 5-31.

C. Meier, Die Entstehung des Politischen bei den Griechen, Frankfurt a. M. 1980.

K. Meuli, Charivari, in: H. Kusch (Hrsg.), Festschrift Franz Dornseiff zum 65. Geburtstag, Leipzig 1953, 231-243 (ND in:

Gesammelte Schriften, Band 1, hrsg. von T. Gelzer, Basel und Stuttgart 1975, 471-484).

O. Meynersen, Der Manteldiebstahl des Sokrates (Ar. Nub. 175-9), Mnemosyne 46, 1993, 18-32. 
Moog-Grünewald 2002

Most 1999

Münz 1998

Nestle 1940

Noël 1997

Nussbaum 1980

Pardini 1993

Parsons 1993

Patzer 1993

Reckford 1987

Ricciardelli Apicella 1993

Riu 1999

Romer 1994

Schmid 1948

Schmitt 2002

Schwinge 1977

Segal 1969

Sifakis 1971

Silk 2000
M. Moog-Grünewald (Hrsg.), Das Neue. Eine Denkfigur der Moderne, Heidelberg 2002.

G. W. Most, From Logos to Mythos, in: Buxton 1999, 25-47.

R. Münz, Theatralität und Theater. Zur Historiographie von Theatralitätsgefügen, Berlin 1998.

W. Nestle, Vom Mythos zum Logos. Die Selbstentfaltung des griechischen Denkens von Homer bis auf die Sophistik und Sokrates, Stuttgart 1940, Aalen ${ }^{3} 1966$.

M.-P. Noël, Mots nouveaux et idées nouvelles dans les Nuées d'Aristophane, Ktema 22, 1997, 173-184.

M. Nussbaum, Aristophanes and Socrates on Learning Practical Wisdom, Yale Classical Studies 26, 1980, 43-97.

A. Pardini, L'ornitogonia (Ar. Av. 693 sgg.) tra serio e faceto: premessa letteraria al suo studio storico-religioso, in: A. Masarcchia (Hrsg.), Orfeo e l'orfismo, Roma 1993, 53-65.

P. Parsons, Identities in Diversity, in: A. Bulloch, E. S. Gruen, A. A. Long und A. Stewart (Hrsg.), Images and Ideologies. SelfDefinition in the Hellenistic World (Hellenistic Culture and Society 12), Berkeley, Los Angeles und London 1993, 152-170.

A. Patzer, Die Wolken des Aristophanes als philosophiegeschichtliches Dokument, in: P. Neukam (Hrsg.), Motiv und Motivation (Dialog-Schule-Wissenschaft. Klassische Sprachen und Literaturen 27), München 1993, 72-93.

K. J. Reckford, Aristophanes' Old-and-New Comedy, Band 1, Chapel Hill und London 1987.

G. Ricciardelli Apicella, Le teogonie orfiche nell'ambito delle teogonie greche, in: A. Masarcchia (Hrsg.), Orfeo e l'orfismo, Roma 1993, 27-51.

X. Riu, Dionysism and Comedy, Lanham, Boulder, New York und Oxford 1999.

F. E. Romer, Atheism, Impiety and the limos mêlios in Aristophanes' Birds, American Journal of Philology 115, 1994, 351-365.

W. Schmid, Das Sokratesbild der Wolken, Philologus 97, 1948, 209-228.

A. Schmitt, Die ,Wende des Denkens auf sich selbst' und die Radikalisierung des Begriffs des Neuen in der Neuzeit, in: Moog-Grünewald 2002, 13-38.

E. R. Schwinge, Aristophanes und die Utopie, Würzburger Jahrbücher 3, 1977, 43-67.

C. P. Segal, Aristophanes' Cloud-Chorus, Arethusa 2, 1969, 143-161 (zitiert nach ND in: H.-J. Newiger (Hrsg.), Aristophanes und die Alte Komödie [Wege der Forschung 265], Darmstadt 1975, 174-197).

G. M. Sifakis, Parabasis and Animal Choruses. A Contribution to the History of Attic Comedy, London 1971.

M. S. Silk, Aristophanes and the Definition of Comedy, Oxford 2000 . 
Strauss 1966

van Gennep 1909

Versnel 1993

Versnel 1999

Walsh 1981

West 1994

Willi 2003

Zannini Quirini 1987

Zimmermann 1983
L. Strauss, Socrates and Aristophanes, New York 1966.

A. van Gennep, Les rites de passage. Étude systématique des rites, Paris 1909 (dt. Übergangsriten, übersetzt von K. Schomburg und S. M. Schomburg-Scherff. Mit einem Nachwort von S. M. Schomburg-Scherff, Frankfurt a. M. 1986).

H. S. Versnel, Transition and Reversal in Myth and Ritual. Inconsistencies in Greek and Roman Religion, Band 2 (Studies in Greek and Roman Religion 6, II), Leiden, New York und Köln 1993.

H. S. Versnel, Komödie, Utopie und verkehrte Welt, in: G. Binder und B. Effe (Hrsg.), Das antike Theater. Aspekte seiner Geschichte, Rezeption und Aktualität, Trier 1999, 93-114.

J. A. Walsh, The Omitted Date in the Athenian Hollow Month, Zeitschrift für Papyrologie und Epigraphik 41, 1981, 107-124.

M. L. West, Orpheus, Sanchuniathon, and the Origins of the Ionian World Model, Classical Quarterly 44, 1994, 289-307.

A. Willi, The Languages of Aristophanes. Aspects of Linguistic Variation in Classical Attic Greek, Oxford 2003.

B. Zannini Quirini, Nephelokokkygia: La prospettiva mitica degli Uccelli di Aristofane (Storia delle Religioni 5), Roma 1987.

B. Zimmermann, Utopisches und Utopie in den Komödien des Aristophanes, Würzburger Jahrbücher 9, 1983, 57-77 (in der überarbeiteten italienischen Fassung „Nephelokokkygia. Riflessioni sull'utopia comica“, in: W. Rösler und B. Zimmermann, Carnevale e utopia nella Grecia antica [Le Rane, Studi 8], Bari 1991, 53-101). 